\title{
Sur une généralisation des coefficients binomiaux
}

\author{
Frédéric Jouhet, Bodo Lass et Jiang Zeng \\ Institut Girard Desargues, Université Claude Bernard (Lyon 1) \\ 43, bd du 11 Novembre 1918, 69622 Villeurbanne cedex, France \\ \{jouhet, lass, zeng\}@igd.univ-lyon1.fr
}

Submitted: Jun 16, 2003; Accepted: Jul 16, 2004; Published: Jul 26, 2004

\begin{abstract}
We prove a recent conjecture of Lassalle about positivity and integrality of coefficients in some polynomial expansions. We also give a combinatorial interpretation of those numbers. Finally, we show that this question is closely related to the fundamental problem of calculating the linearization coefficients for binomial coefficients.
\end{abstract}

\section{Key words}

Positivity and integrality conjectures, linearization coefficients, calculus of finite differences

\section{AMS subject classifications}

05A05, 05A10, 05A15, 05A17, 05A18, 05A19, 05E05, 33C20

\section{Introduction}

Une partition $\mu=\left(\mu_{1} \geq \mu_{2} \geq \cdots \geq \mu_{l}>0\right)$ de $n$ est une suite décroissante d'entiers strictement positifs de somme $n=|\mu|$. Le nombre $l=l(\mu)$ est appelé la longueur de $\mu$. Pour tout $i \geq 1$, l'entier $m_{i}(\mu)=\operatorname{card}\left\{j: \mu_{j}=i\right\}$ est la multiplicité de $i$ dans $\mu$. Définissons

$$
z_{\mu}=\prod_{i \geq 1} i^{m_{i}(\mu)} m_{i}(\mu) !
$$

Pour $n \geq 1$ les factorielles montantes et descendantes sont définies comme suit :

$$
(x)_{n}=x(x+1) \cdots(x+n-1), \quad\langle x\rangle_{n}=x(x-1) \cdots(x-n+1) .
$$

Notons que $\langle-x\rangle_{n}=(-1)^{n}(x)_{n}$ et que les coefficients binomiaux valent $\left(\begin{array}{l}x \\ n\end{array}\right)=\langle x\rangle_{n} / n$ !. Dans ses travaux sur les polynômes de Jack [13] Lassalle a récemment posé la conjecture suivante. 
Conjecture 1. Soit $X$ une indéterminée, $m$ et $n$ deux entiers strictement positifs et $\mathbf{r}=\left(r_{1}, \ldots, r_{m}\right)$ une suite d'entiers positifs telle que $|\mathbf{r}|=\sum_{i=1}^{m} r_{i}>0$. On a

$$
\sum_{|\mu|=n} \frac{X^{l(\mu)-1}}{z_{\mu}}\left(\sum_{i=1}^{l(\mu)} \prod_{k=1}^{m} \frac{\left(\mu_{i}\right)_{r_{k}}}{r_{k} !}\right)=\frac{1}{|\mathbf{r}|} \sum_{k=1}^{\min (n,|r|)} c_{k}^{(\mathbf{r})}\left(\begin{array}{c}
X+n-1 \\
n-k
\end{array}\right)
$$

où les coefficients $c_{k}^{(\mathbf{r})}$ sont des entiers positifs à déterminer.

Remarquons d'abord que le membre de gauche de (1) est un polynôme en $X$ de degré $n-1$, donc il peut être développé dans la base $\left\{\left(\begin{array}{c}X+n-1 \\ n-k\end{array}\right)\right\}(1 \leq k \leq n)$ d'une seule façon. Ceci implique l'existence et l'unicité des coefficients rationnels $c_{k}^{(\mathbf{r})}$ au membre de droite de $(1)$.

Comme nous allons le démontrer, les nombres $c_{k}^{(\mathbf{r})}$ sont en fait des entiers positifs et indépendants de $n$. Pour $m=1$ et $m=2$ les coefficients $c_{k}^{(\mathbf{r})}$ ont été déterminés et la conjecture a été vérifiée (voir $[7,12,13,17])$. Dans le premier cas, on a $c_{k}^{\left(r_{1}\right)}=\left(\begin{array}{c}r_{1} \\ k\end{array}\right)$ et dans le deuxième cas Lassalle [13] a obtenu plusieurs formules exprimant $c_{k}^{\left(r_{1}, r_{2}\right)}$, qui se réduisent au cas précédent lorsque $r_{2}=0$. Donc les coefficients $c_{k}^{(\mathbf{r})}$ sont des extensions des coefficients binomiaux classiques.

L'objectif de cet article est de donner une solution complète de ce problème, ceci par trois approches distinctes utilisant des techniques complètement différentes. Plus précisément, la section 2 donne une réponse analytique à la conjecture 1 , ainsi que quelques identités du même type, ceci à l'aide des fonctions génératrices multivariées. Dans la troisième section, nous donnons une interprétation combinatoire de l'identité suivante :

$$
\sum_{|\mu|=n} \frac{n !}{z_{\mu}} X^{l(\mu)-1} \sum_{i=1}^{l(\mu)} \prod_{k=1}^{m} \mu_{i}\left(\begin{array}{c}
\mu_{i}+r_{k}-1 \\
r_{k}-1
\end{array}\right)=\frac{\prod_{j} r_{j}}{|\mathbf{r}|} \sum_{k=1}^{\min (n,|r|)} c_{k}^{(\mathbf{r})} k !\left(\begin{array}{l}
n \\
k
\end{array}\right)(X+k)_{n-k}
$$

qui est l'identité (1) au facteur $n ! r_{1} \ldots r_{m}$ près. Dans la dernière section, nous détaillons une troisième démonstration de la conjecture de Lassalle qui utilise le calcul aux différences et le cas particulier $m=1$ de (2), c'est-à-dire l'identité :

$$
\sum_{|\mu|=n} \frac{n !}{z_{\mu}} X^{l(\mu)-1} \sum_{i=1}^{l(\mu)} \mu_{i}\left(\begin{array}{c}
\mu_{i}+r_{1}-1 \\
r_{1}-1
\end{array}\right)=\sum_{k \geq 1}\left(\begin{array}{l}
r_{1} \\
k
\end{array}\right) k !\left(\begin{array}{l}
n \\
k
\end{array}\right)(X+k)_{n-k}=\left(X+r_{1}\right)_{n}-(X)_{n}
$$

dont la démonstration est facile, voir [12] pour une preuve algébrique et [17] pour une preuve combinatoire. Dans ce paragraphe, nous voyons que le problème essentiel soulevé par la conjecture de Lassalle est le calcul de certains coefficients de linéarisation. Malgré l'importance fondamentale de cette question, il semble que, jusqu'à présent, les coefficients de linéarisation ne furent étudiés que pour les polynômes orthogonaux. C'est pourquoi nous ajoutons un traitement combinatoire du problème dans cette section. 
Afin de rendre la lecture la plus autonome possible nous rappelons ici quelques formules fréquemment utilisées dans la suite. D'abord la formule binomiale peut s'écrire :

$$
(1-x)^{-\alpha}=\sum_{n \geq 0} \frac{(\alpha)_{n}}{n !} x^{n}
$$

Nous aurons aussi besoin de la transformation suivante, qui est un cas limite de la formule de Whipple [1, p. 142] :

$$
{ }_{3} F_{2}\left[\begin{array}{c}
-n, a, b \\
c, d
\end{array} ; 1\right]=\frac{(c-a)_{n}}{(c)_{n}}{ }_{3} F_{2}\left[\begin{array}{c}
-n, a, d-b \\
d, a+1-n-c
\end{array} ; 1\right],
$$

et qui se réduit à la formule de sommation de Chu-Vandermonde lorsque $b=d$ :

$$
{ }_{2} F_{1}\left[\begin{array}{c}
-n, a \\
c
\end{array} ; 1\right]=\frac{(c-a)_{n}}{(c)_{n}}
$$

où

$$
{ }_{p} F_{q}\left[\begin{array}{c}
a_{1}, a_{2}, \ldots, a_{p} \\
b_{1}, b_{2}, \ldots, b_{q}
\end{array} ; z\right]=\sum_{k \geq 0} \frac{\left(a_{1}\right)_{k} \cdots\left(a_{p}\right)_{k}}{\left(b_{1}\right)_{k} \cdots\left(b_{q}\right)_{k}} \frac{z^{k}}{k !} .
$$

est la définition des fonctions hypergéométriques classiques.

\section{Fonctions génératrices}

En multipliant le membre de gauche de (1) par $t^{n} x_{1}^{r_{1}} \cdots x_{m}^{r_{m}}$ et en sommant sur $n \geq 1$ et les entiers $r_{1}, \ldots, r_{m} \geq 0$ tels que $|\mathbf{r}| \neq 0$, par la formule binomiale (4), nous sommes amenés à évaluer l'expression

$$
\sum_{|\mu| \geq 1} t^{|\mu|} \frac{X^{l(\mu)-1}}{z_{\mu}} \sum_{i=1}^{l(\mu)}\left(\prod_{l=1}^{m}\left(1-x_{l}\right)^{-\mu_{i}}-1\right) .
$$

Lemme 1. Soit y une indéterminée, alors

$$
\sum_{|\mu| \geq 1} t^{|\mu|} \frac{X^{l(\mu)-1}}{z_{\mu}} \sum_{i=1}^{l(\mu)}\left(y^{\mu_{i}}-1\right)=\sum_{n \geq 1} t^{n} \sum_{k=1}^{n}\left(\begin{array}{c}
X+n-1 \\
n-k
\end{array}\right) \frac{(y-1)^{k}}{k} .
$$

Preuve. Toute partition $\mu$ non nulle correspond de façon biunivoque à une suite non nulle 
à support fini $\mathbf{m}=\left(m_{1}, m_{2}, \ldots\right)$ telle que $\mu=\left(1^{m_{1}} 2^{m_{2}} \ldots\right)$. On a donc

$$
\begin{aligned}
\sum_{|\mu| \geq 1} t^{|\mu|} \frac{X^{l(\mu)-1}}{z_{\mu}} \sum_{i=1}^{l(\mu)} y^{\mu_{i}} & =\sum_{\mathbf{m}} X^{-1} \prod_{j \geq 1}\left(\frac{X t^{j}}{j}\right)^{m_{j}} \frac{1}{m_{j} !} \sum_{i \geq 1} m_{i} y^{i} \\
& =\sum_{i \geq 1} y^{i}\left(\sum_{m_{i} \geq 0} m_{i}\left(\frac{X t^{i}}{i}\right)^{m_{i}} \frac{X^{-1}}{m_{i} !}\right) \cdot \prod_{j \neq i} \sum_{m_{j} \geq 0}\left(\frac{X t^{j}}{j}\right)^{m_{j}} \frac{1}{m_{j} !} \\
& =\sum_{i \geq 1} \frac{(y t)^{i}}{i} \exp \left(\frac{X t^{i}}{i}\right) \prod_{j \neq i} \exp \left(\frac{X t^{j}}{j}\right) \\
& =(1-t)^{-X} \log (1-y t)^{-1}
\end{aligned}
$$

Par soustraction du terme correspondant à $y=1$, nous obtenons

$$
\begin{aligned}
\sum_{|\mu| \geq 1} t^{|\mu|} \frac{X^{l(\mu)-1}}{z_{\mu}} \sum_{i=1}^{l(\mu)}\left(y^{\mu_{i}}-1\right) & =(1-t)^{-X} \log \left(1-\frac{t}{1-t}(y-1)\right)^{-1} \\
& =\sum_{k \geq 1}(1-t)^{-X-k} \frac{t^{k}(y-1)^{k}}{k} \\
& =\sum_{n \geq 1} t^{n} \sum_{k=1}^{n}\left(\begin{array}{c}
X+n-1 \\
n-k
\end{array}\right) \frac{(y-1)^{k}}{k}
\end{aligned}
$$

ce qui achève la démonstration.

Notons, pour toute fonction multivariée $f$, par $\left[x_{1}^{r_{1}} \cdots x_{m}^{r_{m}}\right] f\left(x_{1}, \ldots, x_{m}\right)$ le coefficient de $x_{1}^{r_{1}} \cdots x_{m}^{r_{m}}$ dans $f$. Nous déduisons donc de $(7)$, en posant $y=1 /\left(1-x_{1}\right)\left(1-x_{2}\right) \cdots(1-$ $x_{m}$ ), le résultat suivant.

Théorème 1. Soient $c_{k}^{(\mathbf{r})}$ les nombres rationnels définis par (1). Alors

$$
\frac{c_{k}^{(\mathbf{r})}}{|\mathbf{r}|}=\left[x_{1}^{r_{1}} \cdots x_{m}^{r_{m}}\right] \frac{1}{k}\left(\frac{1}{\left(1-x_{1}\right) \cdots\left(1-x_{m}\right)}-1\right)^{k} .
$$

En particulier, $k c_{k}^{(\mathbf{r})} /|\mathbf{r}|$ est un entier positif et ne dépend pas de $n$.

Il en résulte que

$$
\begin{aligned}
c_{k}^{(\mathbf{r})} & =\left.\left[x_{1}^{r_{1}} \cdots x_{m}^{r_{m}}\right] \frac{d}{d z}\right|_{z=1} \frac{1}{k}\left(\frac{1}{\left(1-z x_{1}\right) \cdots\left(1-z x_{m}\right)}-1\right)^{k} \\
& =\left[x_{1}^{r_{1}} \cdots x_{m}^{r_{m}}\right]\left(\frac{1}{\left(1-x_{1}\right) \cdots\left(1-x_{m}\right)}-1\right)^{k-1} \frac{\frac{x_{1}}{1-x_{1}}+\cdots+\frac{x_{m}}{1-x_{m}}}{\left(1-x_{1}\right) \cdots\left(1-x_{m}\right)} .
\end{aligned}
$$

La dernière expression montre clairement le corollaire suivant. 
Corollaire 1. Les nombres $c_{k}^{(\mathbf{r})}$ sont des entiers positifs.

Il est aussi possible de déduire le corollaire au moyen des fonctions symétriques homogènes sur $\left\{x_{1}, \ldots, x_{m}\right\}$, qui sont définies [10,14] par la fonction génératrice :

$$
\sum_{n \geq 0} h_{n}\left(x_{1}, \ldots, x_{m}\right) z^{n}=\prod_{i=1}^{m}\left(1-z x_{i}\right)^{-1},
$$

et donc ceci, à l'aide de (10), permet d'écrire :

$$
\begin{aligned}
& \sum_{k \geq 1} \sum_{r_{1}, \ldots, r_{m} \geq 0} c_{k}^{(\mathbf{r})} t^{k} x_{1}^{r_{1}} \cdots x_{m}^{r_{m}} \\
= & -\left.\frac{d}{d z}\right|_{z=1} \log \left(1-t \sum_{n \geq 1} h_{n}\left(x_{1}, \ldots, x_{m}\right) z^{n}\right) \\
= & \sum_{\lambda}|\lambda| \frac{t^{l(\lambda)}}{l(\lambda)}\left(\begin{array}{c}
l(\lambda) \\
m_{1}(\lambda), m_{2}(\lambda), \ldots
\end{array}\right) h_{\lambda}\left(x_{1}, \ldots, x_{m}\right) \\
= & \sum_{\lambda} t^{l(\lambda)} \sum_{i \geq 1} i\left(\begin{array}{c}
l(\lambda)-1 \\
m_{1}(\lambda), m_{2}(\lambda), \ldots, m_{i}(\lambda)-1, \ldots
\end{array}\right) h_{\lambda}\left(x_{1}, \ldots, x_{m}\right),
\end{aligned}
$$

ce qui montre aussi que $c_{k}^{(\mathbf{r})} \in \mathbb{N}$. Notons que le membre de droite de (11) s'apparente au développement de la nième fonction symétrique puissance $p_{n}\left(x_{1}, \ldots, x_{m}\right)$ dans la base des fonctions symétriques homogènes donné par la formule de Waring [10, 15].

D'autre part, en développant le membre de droite de (9) par la formule binomiale, nous obtenons

$$
\begin{aligned}
& \frac{(-1)^{k}}{k}+\frac{1}{k} \sum_{i \geq 1}(-1)^{k-i}\left(\begin{array}{c}
k \\
i
\end{array}\right)\left(1-x_{1}\right)^{-i} \cdots\left(1-x_{m}\right)^{-i} \\
= & \sum_{|\mathbf{r}|>0} \sum_{i \geq 1} \frac{(-1)^{k-i}}{i}\left(\begin{array}{c}
k-1 \\
i-1
\end{array}\right) \prod_{l=1}^{m}\left(\begin{array}{c}
r_{l}+i-1 \\
r_{l}
\end{array}\right) x_{l}^{r_{l}},
\end{aligned}
$$

ce qui donne, en extrayant le coefficient de $x_{1}^{r_{1}} \cdots x_{m}^{r_{m}}$, le résultat suivant.

Corollaire 2. On a la formule explicite pour $c_{k}^{(\mathbf{r})}$ :

$$
\begin{aligned}
c_{k}^{(\mathbf{r})} & =|\mathbf{r}| \sum_{i \geq 1} \frac{(-1)^{k-i}}{i}\left(\begin{array}{c}
k-1 \\
i-1
\end{array}\right) \prod_{l=1}^{m}\left(\begin{array}{c}
r_{l}+i-1 \\
r_{l}
\end{array}\right) \\
& =\sum_{j=1}^{m} \sum_{i=1}^{k}(-1)^{k-i}\left(\begin{array}{c}
k-1 \\
i-1
\end{array}\right)\left(\begin{array}{c}
i+r_{j}-1 \\
r_{j}-1
\end{array}\right) \prod_{l=1, l \neq j}^{m}\left(\begin{array}{c}
r_{l}+i-1 \\
r_{l}
\end{array}\right) .
\end{aligned}
$$


En particulier, pour $m=1$ et $m=2$, la formule (12) permet de retrouver les deux expressions explicites de Lassalle [13]. En fait, pour $m=1$ la formule (9) se réduit directement à

$$
\frac{k}{r_{1}} c_{k}^{\left(r_{1}\right)}=\left[x_{1}^{r_{1}}\right] x_{1}^{k}\left(1-x_{1}\right)^{-k}=\left[x_{1}^{r_{1}}\right] \sum_{l \geq k}\left(\begin{array}{l}
l-1 \\
k-1
\end{array}\right) x_{1}^{l} \Longrightarrow c_{k}^{\left(r_{1}\right)}=\left(\begin{array}{c}
r_{1} \\
k
\end{array}\right) .
$$

Pour $m=2$ la formule (12) s'écrit

$$
\begin{aligned}
c_{k}^{\left(r_{1}, r_{2}\right)} & =\frac{r_{1}+r_{2}}{k} \sum_{i=1}^{k}(-1)^{k-i}\left(\begin{array}{c}
k \\
i
\end{array}\right)\left(\begin{array}{c}
i+r_{1}-1 \\
r_{1}
\end{array}\right)\left(\begin{array}{c}
i+r_{2}-1 \\
r_{2}
\end{array}\right) \\
& =(-1)^{k-1}\left(r_{1}+r_{2}\right)_{3} F_{2}\left[\begin{array}{c}
\left.-k+1, r_{1}+1, r_{2}+1 ; 1\right] \\
2,1
\end{array} ;\right.
\end{aligned}
$$

Appliquons deux fois la formule (5) à l'expression ci-dessus, ce qui donne bien

$$
c_{k}^{\left(r_{1}, r_{2}\right)}=\left(\begin{array}{c}
r_{1}+r_{2} \\
k
\end{array}\right){ }_{3} F_{2}\left[\begin{array}{c}
-k+1,-r_{1},-r_{2} \\
1-r_{1}-r_{2}, 1
\end{array} ; 1\right] .
$$

Remarquons qu'en appliquant une troisième fois (5), on retrouve une autre expression de [13] :

$$
\begin{aligned}
c_{k}^{\left(r_{1}, r_{2}\right)} & =\left(\begin{array}{c}
r_{1}+r_{2} \\
k
\end{array}\right)\left(\begin{array}{c}
r_{1}+r_{2} \\
r_{1}
\end{array}\right){ }_{3} F_{2}\left[\begin{array}{c}
-r_{1},-r_{2}, k-r_{1}-r_{2} \\
1-r_{1}-r_{2},-r_{1}-r_{2}
\end{array} ;\right] \\
& =\sum_{i \geq 0}(-1)^{i}\left(\begin{array}{c}
r_{1}+r_{2}-i \\
k
\end{array}\right) \frac{r_{1}+r_{2}}{r_{1}+r_{2}-i}\left(\begin{array}{c}
r_{1}+r_{2}-i \\
i
\end{array}\right)\left(\begin{array}{c}
r_{1}+r_{2}-2 i \\
r_{1}-i
\end{array}\right) .
\end{aligned}
$$

Enfin, en multipliant le membre de gauche de (1) par $t^{n} x_{1}^{r_{1}} \cdots x_{m}^{r_{m}}$ et en sommant sur $n \geq 1$ et les entiers $r_{1}, \ldots, r_{m} \geq 0$, nous obtenons

$$
\sum_{|\mu| \geq 1} t^{|\mu|} \frac{X^{l(\mu)-1}}{z_{\mu}} \sum_{i=1}^{l(\mu)} \prod_{l=1}^{m}\left(1-x_{l}\right)^{-\mu_{i}}
$$

ce qui peut se développer directement à l'aide de (8) comme suit :

$$
\sum_{n \geq 0} t^{n} \frac{(X)_{n}}{n !} \sum_{k \geq 1} \frac{1}{k}\left(\frac{t}{\left(1-x_{1}\right) \cdots\left(1-x_{m}\right)}\right)^{k}=\sum_{n, k \geq 1} \frac{t^{n}}{k}\left(\begin{array}{c}
X+n-k-1 \\
n-k
\end{array}\right) \prod_{l=1}^{m} \sum_{r_{l} \geq 0} \frac{(k)_{r_{l}}}{r_{l} !} x_{l}^{r_{l}},
$$

et donc nous obtenons l'identité

$$
\sum_{|\mu|=n} \frac{X^{l(\mu)-1}}{z_{\mu}} \sum_{i=1}^{l(\mu)} \prod_{k=1}^{m} \frac{\left(\mu_{i}\right)_{r_{k}}}{r_{k} !}=\sum_{k=1}^{n} \frac{1}{k} \prod_{l=1}^{m}\left(\begin{array}{c}
r_{l}+k-1 \\
r_{l}
\end{array}\right)\left(\begin{array}{c}
X+n-k-1 \\
n-k
\end{array}\right) .
$$

Il est possible d'établir une extension de (15) comme suit. 
Proposition 1. Pour toute partition $\mu$ et tout $p \in \mathbb{N}$, soit $\left\langle\begin{array}{l}\mu \\ p\end{array}\right\rangle$ le nombre de façons de choisir $p$ éléments dans le diagramme de Ferrers de $\mu$, dont au moins un par ligne, alors

$$
\begin{aligned}
& \sum_{|\mu|=n}\left\langle\begin{array}{c}
\mu \\
p
\end{array}\right\rangle \frac{X^{l(\mu)-1}}{z_{\mu}}\left(\sum_{i=1}^{l(\mu)} \prod_{k=1}^{m} \frac{\left(\mu_{i}\right)_{r_{k}}}{r_{k} !}\right) \\
& =\sum_{k=1}^{p} \frac{1}{k}\left(\sum_{j=k}^{n-p+k}\left(\begin{array}{c}
j-1 \\
k-1
\end{array}\right)\left(\begin{array}{c}
n-j-1 \\
p-k-1
\end{array}\right) \prod_{l=1}^{m}\left(\begin{array}{c}
r_{l}+j-1 \\
r_{l}
\end{array}\right)\right)\left(\begin{array}{c}
X+p-k-1 \\
p-k
\end{array}\right) .
\end{aligned}
$$

Preuve. Rappelons la fonction génératrice suivante [10] :

$$
\sum_{p \geq 1}\left\langle\begin{array}{l}
\mu \\
p
\end{array}\right\rangle x^{p}=\prod_{k \geq 1}\left((1+x)^{k}-1\right)^{m_{k}(\mu)} .
$$

Nous pouvons ainsi, comme pour (15), calculer la fonction génératrice du membre de gauche de (16), en le multipliant par $t^{n} x^{p} x_{1}^{r_{1}} \cdots x_{m}^{r_{m}}$ et en sommant sur $n, p \geq 1$ et $r_{1}, \ldots, r_{m} \geq 0$ :

$$
\begin{aligned}
& \sum_{|\mu| \geq 1} t^{|\mu|} \frac{X^{l(\mu)-1}}{z_{\mu}} \sum_{p \geq 1}\left\langle\begin{array}{c}
\mu \\
p
\end{array}\right\rangle x^{p} \sum_{i=1}^{l(\mu)} \prod_{l=1}^{m}\left(1-x_{l}\right)^{-\mu_{i}} \\
& =\left(1-\frac{t x}{1-t}\right)^{-X}\left[\log \left(1-\frac{t}{\left(1-x_{1}\right) \cdots\left(1-x_{m}\right)}\right)-\log \left(1-\frac{t(1+x)}{\left(1-x_{1}\right) \cdots\left(1-x_{m}\right)}\right)\right] .
\end{aligned}
$$

Développons alors cette dernière expression, ce qui donne :

$$
\begin{aligned}
& \sum_{p \geq 0}\left(\frac{t x}{1-t}\right)^{p} \frac{(X)_{p}}{p !} \sum_{j \geq 1} \frac{1}{j}\left(\frac{t}{\left(1-x_{1}\right) \cdots\left(1-x_{m}\right)}\right)^{j}\left((1+x)^{j}-1\right) \\
& =\sum_{j, k \geq 1} \sum_{p \geq 0} \frac{1}{j}\left(\frac{t x}{1-t}\right)^{p} \frac{(X)_{p}}{p !}\left(\frac{t}{\left(1-x_{1}\right) \cdots\left(1-x_{m}\right)}\right)^{j}\left(\begin{array}{l}
j \\
k
\end{array}\right) x^{k} \\
& =\sum_{j, k, p \geq 1} \frac{1}{j}\left(\begin{array}{l}
j \\
k
\end{array}\right)\left(\begin{array}{c}
X+p-k-1 \\
p-k
\end{array}\right) x^{p}\left(\prod_{l=1}^{m} \sum_{r_{l} \geq 0} \frac{(j)_{r_{l}}}{r_{l} !} x_{l}^{r_{l}}\right) t^{p+j-k}(1-t)^{-p+k} .
\end{aligned}
$$

Mais en utilisant la formule binomiale sous la forme :

$$
(1-t)^{-p+k}=\sum_{n \geq 0} \frac{(p-k)_{n}}{n !} t^{n}
$$

en remplaçant $n$ par $n-p-j+k$ et en extrayant le coefficient devant $x^{p} t^{n} x_{1}^{r_{1}} \cdots x_{m}^{r_{m}}$, nous obtenons la fonction génératrice du membre de droite. 
Remarque. Pour $p=n$, l'identité (16) donne bien (15). Lorsque tous les $r_{i}$ sont nuls, le membre de droite de (1) n'a pas de sens. Or il résulte de (7) avec $y=0$ que

$$
(1-t)^{-X} \log (1-t)^{-1}=\sum_{n \geq 1} t^{n} \sum_{k=1}^{n}\left(\begin{array}{c}
X+n-1 \\
n-k
\end{array}\right) \frac{(-1)^{k-1}}{k}
$$

ce qui donne le prolongement suivant de (1) pour $\mathbf{r}=0$ :

$$
\sum_{|\mu|=n} \frac{X^{l(\mu)-1}}{z_{\mu}} l(\mu)=\sum_{k=1}^{n} \frac{(-1)^{k-1}}{k}\left(\begin{array}{c}
X+n-1 \\
n-k
\end{array}\right) .
$$

Cette formule est en fait la dérivée d'une formule de Macdonald [14, p. 26] :

$$
\sum_{|\mu|=n} \frac{X^{l(\mu)}}{z_{\mu}}=\left(\begin{array}{c}
X+n-1 \\
n
\end{array}\right)
$$

\section{Interprétations combinatoires}

Une permutation $\sigma$ de l'ensemble $E=\left\{a_{1}, \ldots, a_{k}\right\}$ est un cycle si $E=\left\{a_{1}, \sigma\left(a_{1}\right), \ldots\right.$, $\left.\sigma^{k-1}\left(a_{1}\right)\right\}$. On note $\sigma=\left(a_{i}, \sigma\left(a_{i}\right), \ldots, \sigma^{k-1}\left(a_{i}\right)\right)$ pour $1 \leq i \leq k$ et on appelle $\sigma$ un cycle de longueur $k$ ou un $k$-cycle et $E$ le support de $\sigma$. Il est d'usage d'identifier $\sigma$ avec son graphe sagittal $G_{\sigma}$, c'est-à-dire, $a_{i} \rightarrow a_{j}$ est un arc de $G_{\sigma}$ si et seulement si $\sigma\left(a_{i}\right)=a_{j}$. Si $\alpha=\left(a_{1}, a_{2}, \ldots, a_{k}\right)$ et $\beta=\left(b_{1}, b_{2}, \ldots, b_{r}\right)$ sont deux cycles de supports disjoints, un mélange de $\alpha$ et $\beta$ est défini comme un cycle $\left(c_{1}, \ldots, c_{k+r}\right)$, où le mot $w=c_{1} \ldots c_{k+r}$ est un réarrangement de

$$
a_{1} a_{2} \ldots a_{k} b_{i} b_{i+1} \ldots b_{r} b_{1} b_{2} \ldots b_{i-1}, \quad i \in\{1, \ldots, r\}
$$

tel que $a_{1} a_{2} \ldots a_{k}$ et $b_{i} b_{i+1} \ldots b_{r} b_{1} b_{2} \ldots b_{i-1}$ sont deux sous-mots de $w$. Géométriquement mélanger deux cycles $\alpha$ et $\beta$ consiste à insérer $\beta$ dans $\alpha$ (ou l'inverse) pour former un nouveau cycle de longueur $k+r$ en gardant la même orientation.

Exemple 1. Soient $\alpha=(1,2,3,4,5,6)$ et $\beta=(a, b, c, d, e)$. Alors $(1, c, 2,3,4, d, e, 5,6, a, b)$ est un mélange de $\alpha$ et $\beta$ (voir l'illustration Figure 1).

Lemme 2. Soient $\alpha$ et $\beta$ deux cycles de supports disjoints et de longueur $k$ et $r$ respectivement. Alors le nombre de mélanges de $\alpha$ et $\beta$ est donné par

$$
F_{k}(r)=\frac{(k+r-1) !}{(k-1) !(r-1) !}=k\left(\begin{array}{c}
k+r-1 \\
r-1
\end{array}\right)=r\left(\begin{array}{c}
k+r-1 \\
k-1
\end{array}\right) .
$$




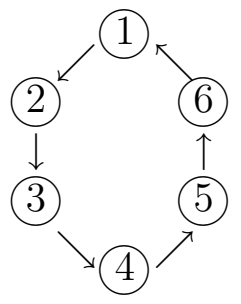

cycle $\alpha$

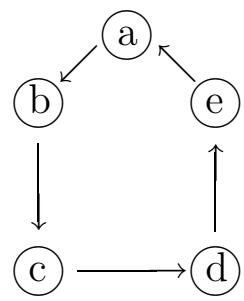

cycle $\beta$

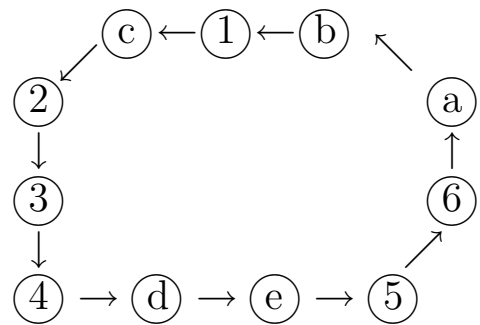

un mélange de $\alpha$ et $\beta$

Figure 1: Un mélange de deux cycles

En effet, il y a $(k+r-1)$ ! manières de constituer un cycle à l'aide de $k+r$ éléments, mais l'ordre des cycles initiaux de longueurs $k$ et $r$ devant être respecté, on obtient le résultat. Remarque. Comme $\left(\begin{array}{c}k+r \\ k\end{array}\right)$ compte le nombre de manières de mélanger deux chemins orientés de longueur $k$ et $r$, respectivement, pour obtenir un chemin orienté de longueur $k+r$, on pourrait appeler $F_{k}(r)$ coefficient binomial cyclique. Or, il semble difficile d'interpréter $F_{k}(r)$ dans le contexte des coefficients binomiaux généralisés de [2]. En effet, $\left(\begin{array}{c}k+r \\ k\end{array}\right)=$ $\left(\begin{array}{c}X^{k+r} \\ X^{k}\end{array}\right)$, où $X^{k}$ est un chemin orienté avec $k$ sommets, mais le coefficient binomial cyclique de [2], à savoir $\left(\begin{array}{c}C_{k+r} \\ C_{k}\end{array}\right)\left(C_{k}\right.$ est un circuit orienté avec $k$ sommets), est égal à 1 si $k$ est un diviseur de $k+r$ et égal à 0 sinon.

La notion de mélange a l'avantage d'être symétrique par rapport aux deux cycles, mais on aura besoin d'une variante asymétrique du mélange dans la suite. Dans un mélange $\gamma$ de deux cycles $\alpha$ et $\beta$, un sommet $a$ de $\alpha$ est $\beta$-décoré par un sommet $b$ de $\beta$ s'il existe un $\operatorname{arc} b \rightarrow a$.

La $\beta$-décoration de $\alpha$ associée à $\gamma$ est le graphe $\gamma^{\prime}$ obtenu en posant $\gamma^{\prime}(b)=\gamma(b)$ pour tout $b$ de $\beta$ ainsi que $\gamma^{\prime}(a)=\alpha(a)$ pour tout $a$ de $\alpha$.

Exemple 2. On reprend l'exemple de la Figure 1. Les éléments 1, 2 et 5 sont décorés par $b, c$ et e respectivement.
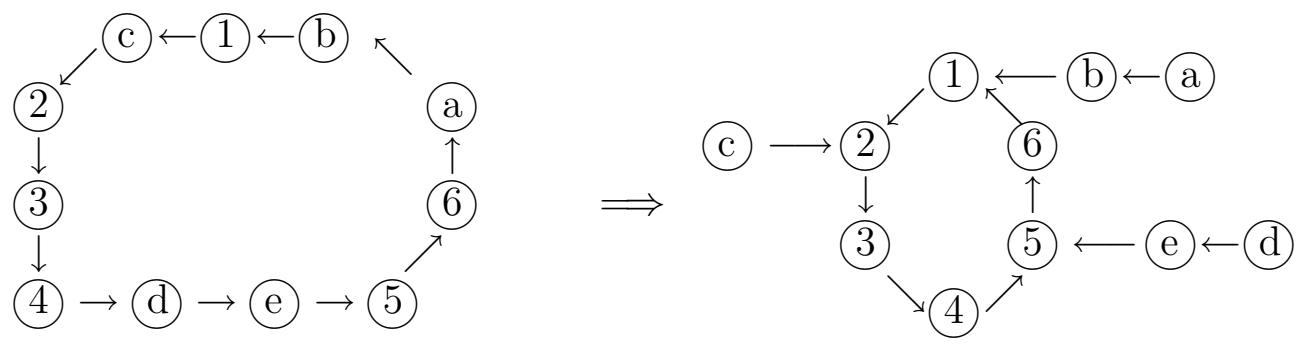

Figure 2: Un mélange de $\alpha$ et $\beta$ et sa $\beta$-décoration de $\alpha$ correspondante 
La notion de décoration permet de donner une autre expression pour $F_{k}(r)$ comme suit :

$$
F_{k}(r)=\sum_{i \geq 1} i\left(\begin{array}{l}
r \\
i
\end{array}\right)\left(\begin{array}{l}
k \\
i
\end{array}\right)
$$

En effet, pour constituer une $\beta$-décoration de $\alpha$ ayant $i$ éléments $\beta$-décorés, on peut d'abord choisir ces éléments dans $\alpha$ de $\left(\begin{array}{c}k \\ i\end{array}\right)$ manières, et puis choisir les $i$ éléments du cycle $\beta$ les décorant de $\left(\begin{array}{l}r \\ i\end{array}\right)$ manières. Il ne reste plus qu'à associer cycliquement ces deux familles de $i$ éléments, ce qui donne $i$ choix, et ceci démontre l'identité ci-dessus.

Remarque. On aurait pu aussi déduire la formule précédente du lemme 2 en partant du membre de droite et en utilisant la formule de Chu-Vandermonde. Inversement on obtient une preuve combinatoire de cette dernière sous la forme suivante :

$$
\sum_{i \geq 1} i\left(\begin{array}{l}
r \\
i
\end{array}\right)\left(\begin{array}{l}
k \\
i
\end{array}\right)=k\left(\begin{array}{c}
k+r-1 \\
r-1
\end{array}\right)=r\left(\begin{array}{c}
k+r-1 \\
k-1
\end{array}\right) .
$$

Considérons maintenant une généralisation de la notion de mélange ou décoration comme suit. Soient $\alpha, \beta_{1}, \ldots, \beta_{m}$ des cycles de supports deux à deux disjoints. On note $\boldsymbol{\beta}=\left(\beta_{1}, \ldots, \beta_{m}\right)$ et on définit une $\boldsymbol{\beta}$-décoration de $\alpha$ comme étant le graphe obtenu en décorant $\alpha$ par chacun de ces $m$ cycles. De plus, on dit qu'une $\boldsymbol{\beta}$-décoration de $\alpha$ est surjective si chaque sommet de $\alpha$ est décoré par au moins un sommet des cycles $\beta_{1}, \ldots, \beta_{m}$.

Exemple 3. Soient $\alpha=(1, \ldots, 6), \beta_{1}=(a, b, c, d, e)$ et $\beta_{2}=(x, y, z, t)$. Considérons les $\beta_{1}$-décoration et $\beta_{2}$-décoration suivantes de $\alpha$ ainsi que la $\left(\beta_{1}, \beta_{2}\right)$-décoration de $\alpha$ correspondante :

On notera que cette décoration est surjective, alors que celle de la Figure 2 ne l'était pas.

Proposition 2. Soient $\alpha, \beta_{1}, \ldots, \beta_{m}$ des cycles de longueur $k, r_{1}, \ldots, r_{m}$ respectivement, et de supports deux à deux disjoints. Si $F_{k}(\mathbf{r})$ (resp. $\left.S_{k}(\mathbf{r})\right)$ est le nombre de $\boldsymbol{\beta}$-décorations (resp. surjectives) de $\alpha$, alors on a

$$
F_{k}(\mathbf{r})=\prod_{i=1}^{m} F_{k}\left(r_{i}\right)=\prod_{l=1}^{m} r_{l}\left(\begin{array}{c}
k+r_{l}-1 \\
r_{l}
\end{array}\right),
$$

et

$$
S_{k}(\mathbf{r})=\sum_{i=1}^{k}(-1)^{k-i}\left(\begin{array}{l}
k \\
i
\end{array}\right) \prod_{l=1}^{m} r_{l}\left(\begin{array}{c}
i+r_{l}-1 \\
r_{l}
\end{array}\right) .
$$

En effet, d'après le lemme 2 la formule (18) est évidente car les $m$ décorations sont indépendantes les unes des autres. D'autre part, il est clair que le nombre de $\boldsymbol{\beta}$-décorations de $\alpha$ ayant $i$ sommets décorés est $\left(\begin{array}{c}k \\ i\end{array}\right) S_{i}(\mathbf{r})$, donc $F_{k}(\mathbf{r})=\sum_{i=1}^{k}\left(\begin{array}{l}k \\ i\end{array}\right) S_{i}(\mathbf{r})$ et par inversion on obtient

$$
S_{k}(\mathbf{r})=\sum_{i=1}^{k}(-1)^{k-i}\left(\begin{array}{l}
k \\
i
\end{array}\right) F_{i}(\mathbf{r}) \text {, }
$$




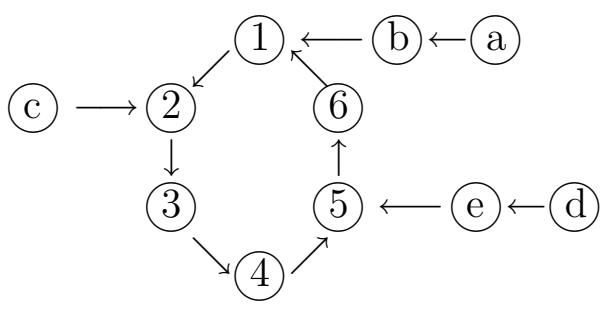

$\beta_{1}$-décoration de $\alpha$

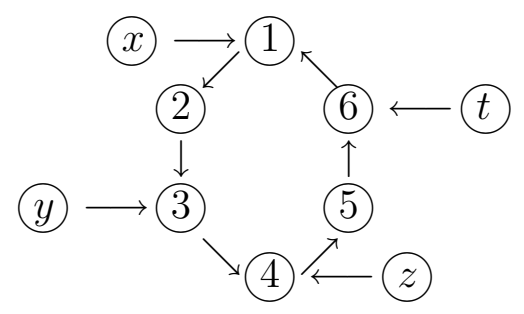

$\beta_{2}$-décoration de $\alpha$

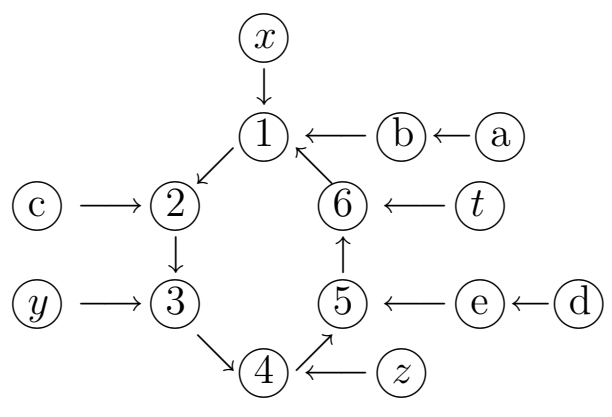

Figure 3: Une $\left(\beta_{1}, \beta_{2}\right)$-décoration de $\alpha$

qui permet de déduire (19) par substitution de (18).

Interprétons maintenant le membre de gauche de (2) à l'aide du modèle précédent. Pour tout entier positif $n$ on note $[n]$ l'ensemble $\{1,2, \ldots, n\}$. Un $\mathcal{L}$-complexe sur $[n]$ est un triplet $(\sigma, \alpha, \boldsymbol{\beta})$, où $\sigma$ est une permutation de $[n], \alpha$ un cycle de $\sigma$ et $\boldsymbol{\beta}$ est une suite de $m$ cycles qui décorent $\alpha$. Étant donnée une partition $\mu$ de $n$, il y a $n ! / z_{\mu}$ permutations de $[n]$ de type $\mu$, c'est-à dire ayant $m_{i}(\mu)$ cycles de longueur $i(1 \leq i \leq n)$. Choisissons un cycle (de longueur $\mu_{i}$ ) à décorer parmi les $l(\mu)$ possibles, les autres cycles étant comptés à l'aide de la variable $X$. La fonction génératrice des $\mathcal{L}$-complexes sur $[n]$ selon le nombre de cycles non décorés est égale à

$$
\sum_{|\mu|=n} \frac{n !}{z_{\mu}} X^{l(\mu)-1} \sum_{i=1}^{l(\mu)} F_{\mu_{i}}(\mathbf{r}) .
$$

D'autre part, on pourrait construire un $\mathcal{L}$-complexe de $[n]$ en constituant d'abord un $k$-cycle à décorer. Il y a $(k-1) !\left(\begin{array}{l}n \\ k\end{array}\right)$ manières différentes de choisir ces éléments, et de les placer sous forme de cycle, qu'on décore ensuite de $F_{k}(\mathbf{r})$ façons (cf. proposition 2). Enfin, comme la fonction génératrice des permutations des $n-k$ éléments restants selon le nombre de cycles est $(X)_{n-k}$, on obtient donc l'identité (15), i.e.,

$$
\sum_{|\mu|=n} \frac{n !}{z_{\mu}} X^{l(\mu)-1} \sum_{i=1}^{l(\mu)} F_{\mu_{i}}(\mathbf{r})=\sum_{k=1}^{n} F_{k}(\mathbf{r})(k-1) !\left(\begin{array}{l}
n \\
k
\end{array}\right)(X)_{n-k} .
$$


Rappelons le résultat suivant, dû à Berge [3], Foata et Strehl [8] (voir aussi [4, p. 91] et $[5,6,11]$ pour d'autres généralisations récentes) :

$$
\sum_{f} X^{\mathrm{cyc} f}=(X+k)_{n-k}
$$

où la somme porte sur toutes les injections $f:[n-k] \rightarrow[n]$ (cyc $f$ est le nombre de cycles de $f$ ). En notant que ces injections peuvent être décomposées en cycles et en $k$ chemins (dont certains peuvent être vides), on peut en présenter une preuve rapide :

$$
\begin{gathered}
\exp \left[X \sum_{i \geq 1}(i-1) ! \frac{t^{i}}{i !}\right]\left[1+\sum_{i \geq 1} i ! \frac{t^{i}}{i !}\right]^{k}=\exp [-X \log (1-t)](1-t)^{-k} \\
=(1-t)^{-X-k}=1+\sum_{i \geq 1}(X+k)_{i} \frac{t^{i}}{i !}
\end{gathered}
$$

Afin d'interpréter le membre de droite de (2) on a besoin d'une notion plus subtile que celle de décoration. Soit $\gamma^{\prime}$ une $\beta$-décoration de $\alpha$. Associons à $\gamma^{\prime}$ son squelette $\gamma^{\prime \prime}$ en posant $\gamma^{\prime \prime}(b)=\gamma^{\prime}(b)$ pour tout $b$ de $\beta$ ainsi que $\gamma^{\prime \prime}(a)=\gamma^{\prime}(a)$ pour tout $a$ de $\alpha$ qui n'est pas $\beta$-décoré. En revanche, si $a$ de $\alpha$ est $\beta$-décoré, alors nous posons $\gamma^{\prime \prime}(a)=\gamma^{\prime p}(a)$ où $p$ est le plus petit entier positif pour lequel $\gamma^{\prime p}(a)$ est $\beta$-décoré.

Exemple 4. On reprend l'exemple de la Figure 2: le squelette obtenu a pour cycle $(1,2,5)$.
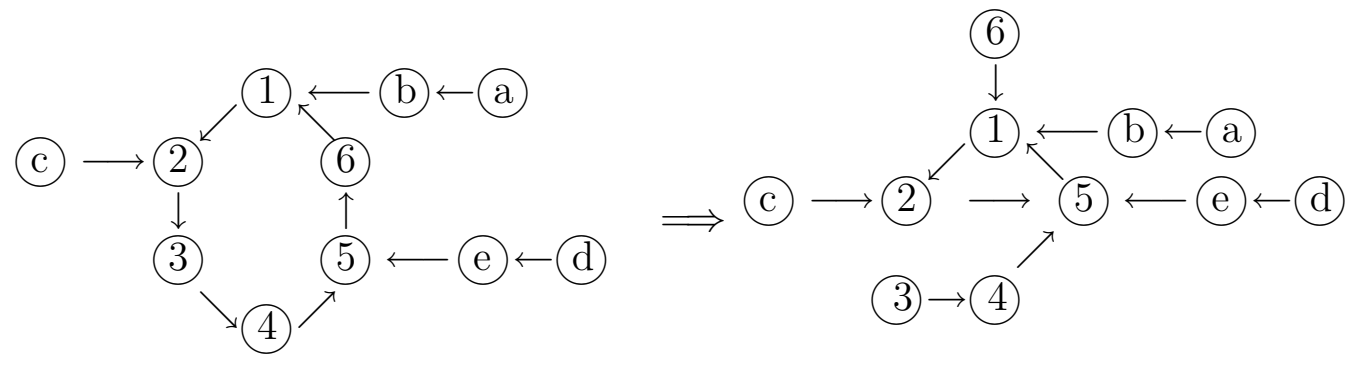

Figure 4: Une $\beta$-décoration de $\alpha$ et son squelette

On définit de façon analogue le squelette d'une $\left(\beta_{1}, \ldots, \beta_{m}\right)$-décoration de $\alpha$, où $\alpha, \beta_{1}, \ldots, \beta_{m}$ sont des cycles de supports deux à deux disjoints et $\beta_{i}$ est de longueur $r_{i}$ pour $1 \leq i \leq m$. Interprétons maintenant le membre de droite de (2). Pour construire un $\mathcal{L}$-complexe sur $[n]$ on peut d'abord former le $k$-cycle $\delta$ du squelette du cycle $\alpha$ décoré. Il y a $(k-1) !\left(\begin{array}{l}n \\ k\end{array}\right)$ manières différentes de former un tel cycle. On décore ensuite $\delta$ de $S_{k}(\mathbf{r})$ façons (cf. proposition 2), car le cycle du squelette est par définition $\boldsymbol{\beta}$-décoré surjectivement. Enfin, comme la fonction génératrice des injections de $n-k$ éléments restant dans les $k$ éléments de $\delta$ selon le nombre de cycles est $(X+k)_{n-k}$ (voir (21)), on a établi le résultat suivant. 
Théorème 2. La fonction génératrice des $\mathcal{L}$-complexes sur $[n]$ selon le nombre de cycles non décorés peut s'exprimer comme suit:

$$
\sum_{|\mu|=n} \frac{n !}{z_{\mu}} X^{l(\mu)-1} \sum_{i=1}^{l(\mu)} F_{\mu_{i}}(\mathbf{r})=\sum_{k=1}^{n} S_{k}(\mathbf{r})(k-1) !\left(\begin{array}{l}
n \\
k
\end{array}\right)(X+k)_{n-k} .
$$

Par comparaison avec (2), on en déduit alors que

$$
c_{k}^{(\mathbf{r})}=\frac{|\mathbf{r}|}{k \cdot \prod_{j} r_{j}} S_{k}(\mathbf{r})=\sum_{j=1}^{m} \frac{S_{k}(\mathbf{r}) \cdot r_{j}}{k \cdot r_{1} \cdots r_{m}},
$$

ce qui montre que $c_{k}^{(\mathbf{r})}$ est positif et ne dépend pas de $n$, et par substitution de (19), on retrouve les formules du corollaire 2 , dont la dernière, à savoir (13), montre que $c_{k}^{(\mathbf{r})}$ est un entier.

En fait, nous pouvons renforcer le dernier résultat, c'est-à-dire la conjecture de Lassalle en supposant que le support de chacun des cycles $\delta, \beta_{1}, \ldots, \beta_{m}$ est totalement ordonné.

Théorème 3. Soit $T_{k}(\mathbf{r} ; j)$ le nombre de $\left(\beta_{1}, \ldots, \beta_{m}\right)$-décorations surjectives de $\delta$ telles que le plus grand élément décorant de $\beta_{j}$ décore le plus grand élément de $\delta$, et le plus grand élément de tout autre cycle décore le plus grand élément de $\delta$ décoré par ce cycle. Alors on a

$$
T_{k}(\mathbf{r} ; j)=\frac{S_{k}(\mathbf{r}) \cdot r_{j}}{k \cdot r_{1} \cdots r_{m}}, \quad \text { pour } \quad 1 \leq j \leq m .
$$

Preuve. Il suffit de regarder l'action de la permutation cyclique $\delta$ ainsi que l'action de la permutation cyclique $\beta_{i}$ pour tout $i \neq j$.

La formule (23) montre par le théorème 3 que nous avons trouvé une interprétation combinatoire pour $c_{k}^{(\mathbf{r})}=\sum_{j=1}^{m} T_{k}(\mathbf{r} ; j)$.

On suppose maintenant que $p$ éléments de $[n]$ sont marqués d'une étoile, dont au moins un par cycle de la permutation de type $\mu$. Ceci donne la fonction génératrice suivante

$$
\sum_{|\mu|=n}\left\langle\begin{array}{c}
\mu \\
p
\end{array}\right) \frac{n !}{z_{\mu}} X^{l(\mu)-1}\left(\sum_{i=1}^{l(\mu)} F_{\mu_{i}}(\mathbf{r})\right) .
$$

On peut d'autre part commencer par choisir les $p$ éléments marqués, et noter $i$ (resp. $j$ ) le nombre d'éléments marqués (resp. non marqués) parmi les $\mu_{i}$ du cycle choisi pour être décoré. Si l'on isole les éléments non marqués de tous les autres cycles, alors la fonction génératrice est

$$
\left(\begin{array}{l}
n \\
p
\end{array}\right) \sum_{i=1}^{p} \sum_{j=0}^{n-p} F_{i+j}(\mathbf{r})(i+j-1) !\left(\begin{array}{c}
p \\
i
\end{array}\right)\left(\begin{array}{c}
n-p \\
j
\end{array}\right)(X)_{p-i} .
$$


Comme il y a $(p-i)(p-i+1)(p-i+2) \cdots(n-i-j-1)=(p-i)_{n-p-j}$ manières différentes de réintroduire les $n-p-j$ éléments restants, on a démontré l'identité suivante

$$
\begin{gathered}
\sum_{|\mu|=n}\left\langle\begin{array}{c}
\mu \\
p
\end{array}\right\rangle \frac{n !}{z_{\mu}} X^{l(\mu)-1}\left(\sum_{i=1}^{l(\mu)} F_{\mu_{i}}(\mathbf{r})\right) \\
=\left(\begin{array}{c}
n \\
p
\end{array}\right) \sum_{i=1}^{p} \sum_{j=0}^{n-p} F_{i+j}(\mathbf{r})(p-i)_{n-p-j}(i+j-1) !\left(\begin{array}{c}
p \\
i
\end{array}\right)\left(\begin{array}{c}
n-p \\
j
\end{array}\right)(X)_{p-i},
\end{gathered}
$$

qui est exactement l'identité (16).

Remarque. Lorsque $m=1$ une preuve analogue de (16) a été donnée dans [17].

\section{Liens avec les coefficients de linéarisation}

Remarquons d'abord qu'en posant $X=0$ dans l'équation (1) nous obtenons

$$
\prod_{i=1}^{m} \frac{(n)_{r_{i}}}{r_{i} !}=\frac{1}{|\mathbf{r}|} \sum_{k=0}^{|\mathbf{r}|} k c_{k}^{(\mathbf{r})} \frac{\langle n\rangle_{k}}{k !} .
$$

Comme $c_{k}^{(\mathbf{r})}$ est indépendant de $n$, la détermination de $c_{k}^{(\mathbf{r})}$ apparaît donc comme le calcul des coefficients du développement du polynôme $(x)_{r_{1}} \cdots(x)_{r_{m}}$ dans la base $\left(\langle x\rangle_{k}\right)_{k \geq 0}$. De plus, si nous pouvons démontrer autrement que les nombres $c_{k}^{(\mathbf{r})}$ sont indépendants de $n$, cette approche fournirait une nouvelle preuve de la conjecture de Lassalle.

Supposons $x$ entier positif et considérons $m$ ensembles $E_{1}, E_{2}, \ldots, E_{m}$, deux à deux disjoints et tels que $\operatorname{card}\left(E_{i}\right)=r_{i}$ pour tout $i \in[m]$. Nous appelons une famille de fonctions $\left(f_{1}, \ldots, f_{m}\right), f_{i}: E_{i} \rightarrow[x]$ pour tout $i \in[m]$, injective si et seulement si chaque fonction $f_{i}$ est injective. Le nombre de familles de fonctions injectives vaut $\langle x\rangle_{r_{1}} \cdots\langle x\rangle_{r_{m}}$. D'autre part, nous pouvons poser $E=E_{1} \cup \cdots \cup E_{m}$ et faire correspondre, de façon bijective, à chaque famille de fonctions injectives une fonction $f: E \rightarrow[x]$ telle que, pour tout $j \in[x]$ et tout $i \in[m], \operatorname{card}\left(f^{-1}(j) \cap E_{i}\right) \in\{0,1\}$. Appelons de manière générale un sous-ensemble $T \subseteq E$ transversal si card $\left(T \cap E_{i}\right) \in\{0,1\}$ pour tout $i \in[m]$. Ceci démontre le théorème suivant.

Théorème 4. Soit $d_{k}\left(r_{1}, \ldots, r_{m}\right)$ le nombre de manières différentes de partitionner $E$ en $k$ transversaux non-vides, alors

$$
\langle x\rangle_{r_{1}} \cdots\langle x\rangle_{r_{m}}=\sum_{k \geq 0} d_{k}(\mathbf{r})\langle x\rangle_{k} .
$$

En particulier, nous avons la formule de linéarisation classique:

$$
\langle x\rangle_{r_{1}}\langle x\rangle_{r_{2}}=\sum_{k \geq 0}\left(\begin{array}{c}
r_{1} \\
k
\end{array}\right)\left(\begin{array}{c}
r_{2} \\
k
\end{array}\right) k !\langle x\rangle_{r_{1}+r_{2}-k} .
$$


En effet, pour $m=2$, s'il y a $k$ transversaux de cardinal deux et si le nombre total de transversaux vaut $r_{1}+r_{2}-k$, alors nous pouvons les choisir de $\left(\begin{array}{c}r_{1} \\ k\end{array}\right)\left(\begin{array}{c}r_{2} \\ k\end{array}\right) k$ ! façons distinctes, c'est-à-dire

$$
d_{r_{1}+r_{2}-k}\left(r_{1}, r_{2}\right)=\left(\begin{array}{c}
r_{1} \\
k
\end{array}\right)\left(\begin{array}{c}
r_{2} \\
k
\end{array}\right) k !
$$

Il est encore plus simple de choisir directement, de façon indépendante, $m$ sousensembles de $[x]$ de cardinaux $r_{1}, \ldots, r_{m}$, respectivement. Ceci est possible de $\left(\begin{array}{c}x \\ r_{1}\end{array}\right) \cdots\left(\begin{array}{c}x \\ r_{m}\end{array}\right)$ manières distinctes et montre le théorème suivant.

Théorème 5. Soit $\tilde{d}_{k}(\mathbf{r})$ le nombre de manières différentes de choisir $m$ sous-ensembles de $[k]$ de cardinaux $r_{1}, \ldots, r_{m}$, respectivement, de sorte que chaque élément de $[k]$ soit choisi au moins une fois. Alors

$$
\left(\begin{array}{c}
x \\
r_{1}
\end{array}\right) \cdots\left(\begin{array}{c}
x \\
r_{m}
\end{array}\right)=\sum_{k \geq 0} \tilde{d}_{k}(\mathbf{r})\left(\begin{array}{l}
x \\
k
\end{array}\right), \quad \tilde{d}_{k}(\mathbf{r})=\frac{k ! d_{k}(\mathbf{r})}{r_{1} ! \cdots r_{m} !} .
$$

En particulier, on a

$$
\tilde{d}_{r_{1}+r_{2}-k}\left(r_{1}, r_{2}\right)=\left(\begin{array}{c}
r_{1}+r_{2}-k \\
k, r_{1}-k, r_{2}-k
\end{array}\right) .
$$

On peut aussi donner une preuve directe de ce dernier résultat. En effet, choisir deux sous-ensembles $E_{1}$ et $E_{2}$ de $[x]$ tels que $\left|E_{1}\right|=r_{1},\left|E_{2}\right|=r_{2}$ et $\left|E_{1} \cap E_{2}\right|=k$ équivaut à choisir un sous-ensemble de $[x]$ de cardinal $r_{1}+r_{2}-k$ et puis le partitionner en trois blocs de cardinaux $k, r_{1}-k, r_{2}-k$, respectivement. D'où $\tilde{d}_{r_{1}+r_{2}-k}\left(r_{1}, r_{2}\right)=\left(\begin{array}{c}r_{1}+r_{2}-k \\ k, r_{1}-k, r_{2}-k\end{array}\right)$.

$\mathrm{Au}$ lieu de choisir, de façon indépendante, $r_{1}, \ldots, r_{m}$ éléments de $[x]$ sans répétition, choisissons-les maintenant avec des répétitions possibles. Comme le nombre de façons de choisir $n$ éléments dans $[x]$ avec des répétitions possibles, c'est-à-dire le nombre de $n$-multi-ensembles sur $[x]$ d'après Stanley $[16$, p. 15], est

$$
\left(\left(\begin{array}{l}
x \\
n
\end{array}\right)\right)=\left(\begin{array}{c}
x+n-1 \\
n
\end{array}\right)=\frac{(x)_{n}}{n !}
$$

le nombre de scénarios distincts est donc égal à $\left(\left(\begin{array}{c}x \\ r_{1}\end{array}\right)\right) \cdots\left(\left(\begin{array}{c}x \\ r_{m}\end{array}\right)\right)$. D'autre part, le nombre de façons de choisir $k$ éléments dans $[x]$ sans répétition est $\left(\begin{array}{l}x \\ k\end{array}\right)$. On en déduit le résultat suivant.

Théorème 6. Soit $\tilde{c}_{k}(\mathbf{r})$ le nombre de manières différentes de choisir $r_{1}, \ldots, r_{m}$ éléments de $[k]$ avec des répétitions possibles, de sorte que chaque élément de [k] soit choisi au moins une fois, alors

$$
\left(\left(\begin{array}{c}
x \\
r_{1}
\end{array}\right)\right) \cdots\left(\left(\begin{array}{c}
x \\
r_{m}
\end{array}\right)\right)=\sum_{k \geq 0} \tilde{c}_{k}(\mathbf{r})\left(\begin{array}{l}
x \\
k
\end{array}\right) .
$$

On en déduit en particulier pour $m=1$

$$
\tilde{c}_{k}\left(r_{1}\right)=\left(\begin{array}{c}
r_{1}-1 \\
r_{1}-k
\end{array}\right)
$$


d'après (28), et pour $m=2$

$$
\tilde{c}_{k}\left(r_{1}, r_{2}\right)=\sum_{k_{1}+k_{2}=k+l}\left(\begin{array}{c}
k \\
l, k_{1}-l, k_{2}-l
\end{array}\right)\left(\begin{array}{c}
r_{1}-1 \\
r_{1}-k_{1}
\end{array}\right)\left(\begin{array}{c}
r_{2}-1 \\
r_{2}-k_{2}
\end{array}\right) .
$$

En effet, supposons que les choix de $r_{1}$ et $r_{2}$ éléments dans $[k]$ avec répétitions possibles ont respectivement $k_{1}$ et $k_{2}$ éléments distincts et $l$ éléments communs. Comme chaque élément de $[k]$ doit être choisi au moins une fois, ceci donne des couples $\left(E_{1}, E_{2}\right)$ de parties de $[k]$ tels que

$$
\left|E_{1}\right|=k_{1}, \quad\left|E_{2}\right|=k_{2}, \quad\left|E_{1} \cap E_{2}\right|=l, \quad E_{1} \cup E_{2}=[k] .
$$

Il y a clairement $\left(\begin{array}{c}k \\ l, k_{1}-l, k_{2}-l\end{array}\right)$ tels couples. Nous appliquons ensuite (30) à $E_{1}$ et $E_{2}$ respectivement, ce qui donne le nombre $\left(\begin{array}{c}r_{1}-1 \\ r_{1}-k_{1}\end{array}\right)\left(\begin{array}{c}r_{2}-1 \\ r_{2}-k_{2}\end{array}\right)$ de choix correspondant au couple $\left(E_{1}, E_{2}\right)$.

Notons que l'identité (26) s'écrit encore

$$
\frac{(x)_{r_{1}}}{r_{1} !} \frac{(x)_{r_{2}}}{r_{2} !}=\sum_{l \geq 0}(-1)^{l}\left(\begin{array}{c}
r_{1}+r_{2}-l \\
l, r_{1}-l, r_{2}-l
\end{array}\right) \frac{(x)_{r_{1}+r_{2}-l}}{\left(r_{1}+r_{2}-l\right) !} .
$$

En reportant (32) dans (1) nous déduisons le résultat suivant.

Lemme 3. Les coefficients $c_{k}^{(\mathbf{r})}$ satisfont la relation de récurrence suivante:

$$
\frac{c_{k}^{\left(r_{1}, r_{2}, r_{3}, \ldots, r_{m}\right)}}{r_{1}+r_{2}+r_{3}+\cdots+r_{m}}=\sum_{l \geq 0}(-1)^{l}\left(\begin{array}{c}
r_{1}+r_{2}-l \\
l, r_{1}-l, r_{2}-l
\end{array}\right) \frac{c_{k}^{\left(r_{1}+r_{2}-l, r_{3}, \ldots, r_{m}\right)}}{r_{1}+r_{2}-l+r_{3}+\cdots+r_{m}}
$$

En particulier, comme $c_{k}^{\left(r_{1}\right)}=\left(\begin{array}{c}r_{1} \\ k\end{array}\right)$ (voir (14)), les coefficients $c_{k}^{(\mathbf{r})}$ sont indépendants de $n$.

En vue de déduire une nouvelle preuve de la conjecture de Lassalle, nous introduisons quelques notations supplémentaires. Pour tout polynôme $p(x)$ définissons les opérateurs $E, I$ et $\Delta$ comme suit :

$$
E p(x)=p(x+1), \quad I p(x)=p(x) \quad \text { et } \quad \Delta=E-I .
$$

Pour tout $k \geq 0$ posons $\Delta^{0}=I$ et $\Delta^{k+1}=\Delta\left(\Delta^{k}\right)$. La formule binomiale implique alors que

$$
\Delta^{n} p(x)=(E-I)^{n} p(x)=\sum_{k=0}^{n}(-1)^{k}\left(\begin{array}{l}
n \\
k
\end{array}\right) p(x+n-k),
$$

et d'autre part nous avons le développement de Taylor suivant :

$$
p(x)=\sum_{k \geq 0} \frac{\Delta^{k} p(0)}{k !}\langle x\rangle_{k}
$$


En appliquant (34) et (35) au polynôme $p(x)=(x)_{r_{1}} \cdots(x)_{r_{m}}$ nous obtenons

$$
\prod_{i=1}^{m}(x)_{r_{i}}=\sum_{k=0}^{|\mathbf{r}|}\left(\frac{1}{k !} \sum_{j=0}^{k}(-1)^{j}\left(\begin{array}{c}
k \\
j
\end{array}\right) \prod_{i=1}^{m}(k-j)_{r_{i}}\right)\langle x\rangle_{k} .
$$

Grâce au lemme 3 la comparaison de (24) avec (29) et (36) montre le théorème suivant.

Théorème 7. On a d'une part

$$
c_{k}^{(\mathbf{r})}=\frac{r_{1}+\cdots+r_{m}}{k} \tilde{c}_{k}(\mathbf{r})
$$

et d'autre part la formule explicite (13), c'est-à-dire,

$$
c_{k}^{(\mathbf{r})}=\sum_{j=1}^{m} \sum_{i=1}^{k}(-1)^{k-i}\left(\begin{array}{c}
k-1 \\
i-1
\end{array}\right)\left(\begin{array}{c}
i+r_{j}-1 \\
r_{j}-1
\end{array}\right) \prod_{l=1, l \neq j}^{m}\left(\begin{array}{c}
r_{l}+i-1 \\
r_{l}
\end{array}\right) .
$$

Il résulte respectivement de (38) et (37) que $c_{k}^{(\mathbf{r})}$ est entier et positif.

Remarque. Il est évident que (29) et (9) fournissent exactement les mêmes interprétations combinatoires pour les nombres $c_{k}^{(\mathbf{r})}$. Enfin, des $q$-analogues naturels de ces coefficients ont été introduits dans [9].

Remerciements. Les auteurs remercient Pierre Leroux ainsi que les deux rapporteurs anonymes pour leurs conseils avisés concernant la rédaction de la troisième section.

\section{BIBLIOGRAPHIE}

[1] G. Andrews, R. Askey et R. Roy, Special Functions, Encyclopedia of Math. and its Applications, 71 (2000).

[2] P. Auger, G. Labelle et P. Leroux, Generalized binomial coefficients for molecular species, Journal of Combinatorial Theory, Series A, Special Issue dedicated to the Memory of Professor G.-C. Rota, 91 (2000), 15-48.

[3] C. Berge, Chemins hamiltoniens, ICC Research Report nº 67/2 (1967).

[4] F. Bergeron, G. Labelle et P. Leroux, Théorie des espèces et combinatoire des structures arborescentes, Publ. LACIM, vol. 19, Montréal (1994).

[5] T. Chow, The path-cycle symmetric function of a digraph, Advances in Mathematics, 118 (1996), 71-98.

[6] F. R. K. Chung et R. L. Graham, On the cover polynomial of a digraph, Journal of Combinatorial Theory, Series B, 65 (1995), 273-290. 
[7] T. Eisenkölbl, Proof of a partition identity conjectured by Lassalle, arXiv:math.CO/9903019.

[8] D. Foata et V. Strehl, Combinatorics of Laguerre polynomials, in Enumeration and design (Waterloo, Ont., 1982), Academic Press, Toronto, ON, 1984, 123-140.

[9] S. J. X. Hou et J. Zeng, Two new families of q-positive integers, arXiv:math.CO/0403064.

[10] F. Jouhet et J. Zeng, Généralisation de formules de type Waring, Séminaire Lotharingien de Combinatoire 44, 2000.

[11] B. Lass, Variations sur le thème $E+\bar{E}=X Y$, Advances in Applied Mathematics, 29 (2002), 215-242.

[12] M. Lassalle, Une identité en théorie des partitions, Journal of Combinatorial Theory, Series A, 89 (2000), 270-288.

[13] M. Lassalle, A new family of positive integers, Ann. Combin. 6 (2002), 399-405.

[14] I. G. Macdonald, Symmetric Functions and Hall Polynomials, Second Edition, Oxford Science Publications, 1995.

[15] M. P. MacMahon, Combinatory analysis, reprinted by Chelsea Publ. Company, 1960.

[16] R. Stanley, Enumerative Combinatorics, vol. 1, Cambridge Studies in Advanced Mathematics 49, Cambridge University Press, 1997.

[17] J. Zeng, A bijective proof of Lassalle's partition identity, Journal of Combinatorial Theory, Series A, 89 (2000), 289-290. 